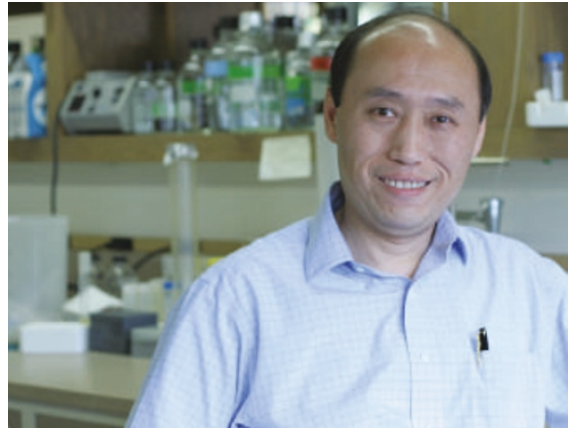

Huimin Kong: using helicase avoids having to put the heat on.

amplicons of anything from 100 to 600 base pairs in 2 to 3 minutes," says Hendrik Viljoen of the department of chemical engineering at the University of Nebraska in Lincoln, one of the designers. "Going faster than 5 minutes doesn't really gain much for the working scientist, since it usually takes longer than that to mix the PCR reagents," says team member Michael Nelson, "so we have backed off on speed and are now primarily concerned with system engineering for reliability and ease of use."

The PCRJet takes eight samples of 20$100 \mathrm{ml}$ at a time. "Talking to people in industry, we have found that in areas like infectious disease detection there is strong resistance to too small volumes. You need a big enough lump of sample because in the early stages of disease development there may be very few organisms present in a sample," says Viljoen. PCRJet needs a fast enzyme, and it uses
KOD Pol from Pyrococcus kodakaraensis from Toyobo Company of Osaka, Japan. Toyobo's Hideki Hayami, who is collaborating with MegaBase, says this can copy DNA at a rate of around 300 nucleotides per second.

Getting personal, Stratagene's Mx3000P is a four-colour optics real-time PCR machine for personal and small lab use, while the 46-well MJ Mini thermal cycler from BioRad of Hercules, California (which recently acquired the manufacturers MJ Research) can be upgraded to a two-colour real-time machine with the retrofit of a MiniOpticon detection system.

\section{Isothermal alternatives}

Given that the physics of heating and cooling a PCR system can be problematic, a few pioneers are developing isothermal procedures - PCR at a uniform temperature - with an eye mainly on the clinical diagnostic market.

One approach is known as the ramification amplifying method (RAM). Invented by David Zhang of Mount Sinai School of Medicine in New York in 1994, RAM is licensed to Hamilton Thorne Biosciences of Beverly, Massachusetts, by Mount Sinai, which was recently granted an additional US patent on the method.

Target DNA is first isolated by capture probes linked to magnetic beads. A given target DNA is then detected by RAM, which employs a single-stranded DNA 'C-probe' that contains $3^{\prime}$ and $5^{\prime}$ sequences complementary to the target. If the $\mathrm{C}$-probe hybridizes accurately with the target DNA, both ends of the probe bind close together and are joined by a ligase to form a circle. Once the circle is formed, this binds a primer at an internal site, which is extended by a strand-displacing DNA polymerase (for example, phi29 or Bst). As the polymerase travels around the circle it displaces the strand created on previous circuits, creating a long chain, which can itself can be duplicated by other primers and enzymes.

As the displaced DNAs are single stranded, primers can bind at a consistent temperature, removing the need for any thermocycling during amplification. However, "circle formation does require annealing of the C-probe to the targets and for long double-stranded DNA targets, a denaturation step would be beneficial," explains David Lane, vice-president of research and development for Hamilton Thorne Biosciences.

Binding is highly specific, making it a useful tool for SNP detection. "Since RAM uses a universal primer, there is also no need for primer balancing, making high-level multiplexing as straightforward as adding multiple C-probes to an assay", says Lane.

A completely different approach is that of Huimin Kong and colleagues, who came up with the idea of using a DNA helicase to separate the DNA strands rather than heat while working at New England Biolabs in Beverly, Massachusetts. "While other so-called isothermal techniques need an initial $95^{\circ} \mathrm{C}$ DNA denaturation step, helicase-dependent amplification (HDA) can be performed in true isothermal conditions," Kong says. Once

\title{
PHOTOCOPIERS FOR DNA
}

Rather than amplifying just the DNA between two primers, many researchers want to amplify entire genomes, to make archive copies of the total DNA from a unique biopsy sample, for example. "If you have a piece of paper with valuable information on it, obviously you don't want to lose the paper, but instead photocopy it many times, store some copies appropriately and use others," says Andy Betera vice-president of product management of GE Healthcare, based in Little Chalfont, UK. Whole-genome amplification (WGA) systems aim to act as photocopiers for DNA. "From the point of view of the molecular epidemiologist or molecular geneticist, the overwhelming potential advantage of the newer methods of WGA is the possibility of producing additional genomic DNA, or amplified WGA DNA, for genotyping, sequencing or other types of genetic analysis like loss of heterozygosity (LOH)," says Andrew Bergen, staff scientist at the National Cancer Institute in Bethesda, Maryland.

"For the past 12 years there have been PCRbased methods of WGA with about a 10 or $15 \%$ locus bias, meaning that 10 or $15 \%$ of the genetic loci in the genome would not be amplified," explains Bergen. Newer methods can avoid this loss in the right conditions. But problems really arise when you have only a very small sample from a slide or biopsy to play with. Bergen says

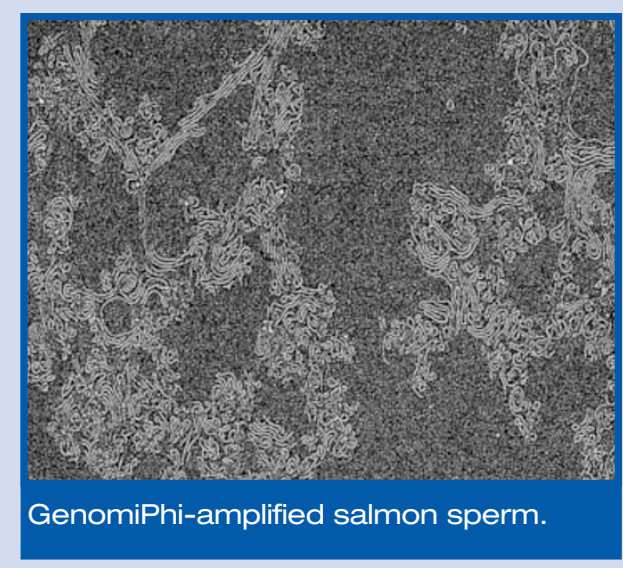

colleagues who have almost exhausted the material from a particular sample ask him: "Can this sample be rescued?" And no WGA method is really effective with less than $10 \mathrm{ng}$ of genomic DNA, he says.

On the commercial front, GE Healthcare's GenomiPhi DNA amplification kit uses the highly processive single-strand displacing DNA polymerase from phage phi29. Short primers are bound randomly throughout the genome and the enzyme copies the DNA, starting from each primer and displacing the primer ahead of it. The result is a soup of genomic fragments of varying lengths, averaging around 10,000 bases. The phi29 method is able to efficiently amplify a whole genome with no loss of sequence if high molecular weight DNA is used as the starting material.

The GenomePlex WGA kit from SigmaAldrich uses technology licensed from Rubicon Genomics of Ann Arbor, Michigan. In this method, the genomic DNA is initially broken up into fragments of around 400 bases long, which are then attached to an identical sequence. The collection is amplified by PCR using a primer that recognizes this sequence. Because short fragments are being copied, this method can cope with starting DNA of high or low molecular weight. 